\title{
Tube Model Predictive Control with an Auxiliary Sliding Mode Controller
}

\author{
Miodrag Spasić ${ }^{1,2}$ Morten Hovd ${ }^{2}{\text { Darko } \text { Mitić }^{1} \text { Dragan Antić }}^{1}$ \\ ${ }^{1}$ Department of Control Systems, Faculty of Electronic Engineering, University of Niš, Serbia. \\ E-mail: \{Miodrag.Spasic, Darko.Mitic, Dragan.Antic\} @elfak.ni.ac.rs \\ ${ }^{2}$ Department of Engineering Cybernetics, Norwegian University of Science and Technology, Trondheim, Norway. \\ E-mail: \{Morten.Hovd, Miodrag.Spasic\} @itk.ntnu.no
}

\begin{abstract}
This paper studies Tube Model Predictive Control (MPC) with a Sliding Mode Controller (SMC) as an auxiliary controller. It is shown how to calculate the tube widths under SMC control, and thus how much the constraints of the nominal MPC have to be tightened in order to achieve robust stability and constraint fulfillment. The analysis avoids the assumption of infinitely fast switching in the SMC controller.
\end{abstract}

Keywords: Model Predictive Control, robustness, Sliding Mode Control, Mixed Integer Linear Program

\section{Introduction}

Model Predictive Control (MPC) has been a great industrial success, particularly in the process industries (Qin and Badgwell, 2003). Still, robustness of MPC controllers continues to be an active research issue. There are many approaches to robust MPC, including dynamic programming, optimization over feedback policies, min-max MPC, etc. Providing an overview over these various approaches to robust MPC is beyond the scope of this paper, the interested reader is instead referred to the bibliographic notes in Chapter 3 of Rawlings and Mayne (2009). As is noted in (Rawlings and Mayne, 2009), optimal or close-tooptimal approaches to robust MPC generally have prohibitive online calculation requirements. More practical approaches are therefore a compromise between performance and online calculation requirements.

A popular approach among such MPC formulations that trade off performance against online calculation requirements is the so-called Tube MPC (Rawlings and Mayne, 2009). In the 'basic' Tube MPC, the MPC controller essentially controls the nominal plant, while there is an auxiliary controller that keeps 'all possible' plants inside a 'tube' close to the nominal plant. To ensure robust constraint satisfaction, the state/output constraints of the nominal MPC have to be modified to account for the width of the tube, and the magnitudes of control inputs that are available to the nominal MPC also have to be reduced to account for the additional input component coming from the auxiliary controller.

It is clearly desirable that the auxiliary controller should add little to the overall computational requirements of the control. If this is the case, the auxiliary controller may execute at a significantly higher sampling frequency than the nominal MPC, thereby taking advantage of feedback also between the samples of the nominal MPC. There is substantial ongoing research effort aiming to reduce the online calculation requirements of MPC. However, approaches like explicit MPC (Bemporad et al., 2002) appear to be limited to a fairly modest number of states, and approaches focusing on online solution of an optimization problem will inevitably have a significant calculation requirement. The higher calculation requirements for MPC will typically result in modest sampling frequencies compared to what is achievable with linear state feedback or controllers of similar complexity. The 
computational requirements of the Sliding Mode Control (SMC) are trivial, and combined with the good robustness properties of SMC this should make SMC an attractive candidate for the auxiliary controller in Tube MPC.

SMC is known for its robustness to parameter variations and external disturbances. It belongs to a special class of nonlinear discontinuous control algorithms, known as variable structure control (Utkin, 1978; Young et al., 1999; Yu and Kaynak, 2009). In its basic form, the SMC input is simply a relay output, depending on the location of the present state relative to a switching surface, which forces a system state to move along this surface, known also as a sliding surface. The modern realization of SMC, by using microcontrollers or digital signal processors, causes a quasi-sliding motion (Milosavljević, 1985) usually in $\mathrm{O}(\mathrm{T})$ vicinity of sliding surface, with $\mathrm{T}$ denoting a sampling time, which could induce a chattering, manifested by high-frequency control signal exciting unmodelled system dynamics and reducing plant lifecycle. An overview of existing digital SMC algorithms is given in (Milosavljević, 2004).

In recent years, researchers have developed several control methodologies based on the combination of SMC and MPC. The combination of SMC and generalized predictive control (GPC), as a subclass of MPC, is discussed in (Corradini and Orlando, 1997; Mitić et al., 2013) for systems with discrete-time transfer function models. In (Garcia-Gabin et al., 2009) the cost function is partially optimized with respect to only the predictive part of controller, while sliding mode control is not involved in the optimization problem. Moreover, the reaching and existence conditions of sliding mode are not derived and the stability issues are not discussed. Unfortunately, all these approaches cannot deal with MIMO systems.

In digital SMC based on state-space models, and hence applicable to MIMO systems, one approach to control law design is to force the system to reach the sliding surface at the very next sampling instant ( $\mathrm{Su}$ et al., 2000). This digital SMC method provides a $O\left(T^{2}\right)$ sliding mode accuracy when the system disturbances are known at each time instant or a unit-step delayed disturbance estimate is used in the presence of unmodeled disturbances. However, when the disturbance depends on the control input, the system becomes unstable or causes chattering. That is why two different approaches in integrating digital SMC and MPC are proposed in (Neelakantan, 2005). The first one applies direct optimization of a cost function criterion with respect to the equivalent control. The second control method splits the controller into the equivalent control part, ensuring the system to stay on sliding sur- face once reached, and the reaching control part that guides the system towards the sliding surface. The cost function is optimized with respect to the latter control term.

In (Incremona et al., 2015; Benattia et al., 2015) hierarchical control schemes, consisting of a high level MPC and a low level SMC (Rubagotti et al., 2011), are considered. The role of the SMC component is to reject the matched disturbances acting the plant, and to reduce uncertainty for the MPC design in that way.

The accurate calculation of the tube widths is complicated even when using linear state feedback. A set of states needs to be calculated, inside which the auxiliary controller is able to keep the states of the real system. Once this set is calculated, the corresponding set inside which the input from the auxiliary control will remain has to be calculated. To avoid restricting the nominal MPC unnecessarily, the calculated set should be as small as possible, i.e, we wish to calculate the socalled minimal robustly positive invariant set (mRPI) for the system under the auxiliary control. Unfortunately, the mRPI will typically be excessively complex and demanding to calculate, but good outer approximations can usually be found when linear state feedback is used as an auxiliary controller (Raković et al., 2005).

To the authors' knowledge, there is no previous work on calculating robustly positively invariant sets for systems under SMC control with a finite sampling frequency for the SMC. However, we will also take an alternative approach: following an idea in Rawlings and Mayne (2009), the robustly positively invariant set will not be calculated directly. Instead, we will show how to calculate, for each constraint, how far in the direction of the constraint the true system can be driven by the model uncertainty. This will give a direct measure of how much each constraint will need to be tightened.

Tube MPC was originally proposed with the MPC handling the nominal system only, and the auxiliary controller handling the deviations from nominal behavior. Alternative formulations have been proposed later, where feedback is introduced also into the MPC part of Tube MPC. In this paper, including the example, we have chosen to use a nominal Tube MPC as in the original Tube MPC formulation, in order to highlight the robustness improvement from the auxiliary controller. However, the SMC-based auxiliary controller proposed in the paper would be equally applicable to a Tube MPC formulation where feedback is used also in the MPC part of the Tube MPC.

The paper is organized as follows. In Section 2, the control problem is introduced. Section 3 briefly describes two proposed digital sliding mode control approaches. The algorithms for calculating the con- 
straints tightening is presented in Section 4. The proposed Tube MPC with an auxiliary SMC has been applied to the real DC servo system (Inteco, 2011), and the digital simulation and experimental results are given in Section 5. Section 6 contains some concluding remarks.

\section{Problem description}

Consider the discrete time system described by the model

$$
x_{k+1}=A x_{k}+B u_{k}+E w_{k},
$$

with the system state $x \in \mathbb{R}^{n_{x}}$, the input $u \in \mathbb{R}^{n_{u}}$, and the disturbance $w \in \mathbb{R}^{n_{w}}$. There are also constraints on the allowable state

$$
x \in \mathbb{X}=\{x \mid F x \leq f\},
$$

constraints on the allowable input

$$
u \in \mathbb{U}=\{u \mid \Gamma u \leq \gamma\},
$$

and constraints on the possible range of disturbances

$$
w \in \mathbb{W}=\{w \mid H w \leq h\} .
$$

It follows from the description above that the sets $\mathbb{X}, \mathbb{U}$, and $\mathbb{W}$ are polyhedral; we will also assume that they are bounded (and thus that the sets are polytopic), of full dimension, and contain the origin in their interior.

In the following, the system state is split into two components, a nominal component $z$ and a deviation from nominal $\epsilon$

$$
x=z+\epsilon .
$$

Similarly, the input is split into the input from the nominal MPC $v$, and the input $\nu$ from the auxiliary controller

$$
u=v+\nu .
$$

The dynamics, described by eq. (1), may therefore be split into the nominal dynamics and the deviation from nominal

$$
\begin{array}{cc}
z_{k+1}= & A z_{k}+B v_{k} \\
\epsilon_{k+1} & =A \epsilon_{k}+B \nu_{k}+E w_{k}
\end{array}
$$

Clearly, eqs. (7) and (8) add to eq. (1). The control scheme is illustrated in Figure 1.

At each timestep, the nominal MPC solves the problem

$$
\begin{aligned}
\min _{\mathbf{z}, \mathbf{v}} J(\mathbf{z}, \mathbf{v}) & \\
& \text { subject to } \\
z_{k+i} \in\left\{z_{k+i} \mid F z_{k+i} \leq f-\delta_{i}^{z}\right\} & i \in\{0,1, \ldots, N\}, \\
v_{k+i} \in\left\{v_{k+i} \mid \Gamma v_{k+i} \leq \gamma-\delta_{i}^{v}\right\} & i \in\{0,1, \ldots, N\} .
\end{aligned}
$$

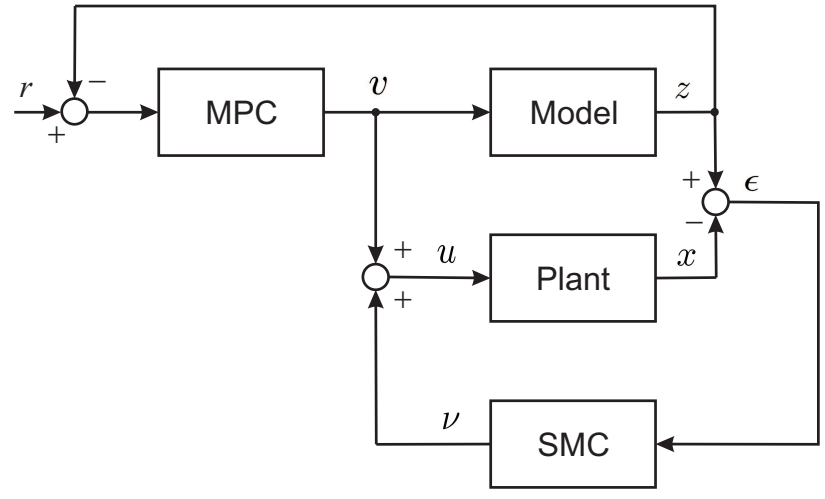

Figure 1: Control scheme

Here, $J(\mathbf{z}, \mathbf{v})$ is the MPC cost function ${ }^{1}, N$ is the length of the prediction horizon for the MPC, $\mathbf{z}$ is the vector of present and future nominal states in the prediction horizon, $\mathbf{z}^{T}=\left[\begin{array}{llll}z_{k}^{T} & z_{k+1}^{T} & \ldots & z_{k+N}^{T}\end{array}\right]$, and $\mathbf{v}$ is the vector of present and future inputs from the nominal MPC in the prediction horizon, $\mathbf{v}^{T}=$ $\left[\begin{array}{llll}v_{k}^{T} & v_{k+1}^{T} & \cdots & v_{k+N}^{T}\end{array}\right]$. The vector $\delta_{i}^{z}$ quantifies how much the nominal state constraints have to be restricted at time $k+i$ in order to ensure that the true state adheres to the original constraints, while the vector $\delta_{i}^{v}$ quantifies how much the constraints on the nominal MPC input have to be restricted at time $k+i$ in order to ensure that the total input adheres to the original constraints. The simplest Tube MPC formulations treat $\delta_{i}^{z}$ and $\delta_{i}^{v}$ as constants over the prediction horizon, whereas other formulations allow these to vary to account for the fact that the disturbance will typically be able to drive the true state further from the nominal state (also under the action of the auxiliary control) over a time period of several timesteps than over a single timestep.

Remark. A special terminal set for the state is a common ingredient in MPC formulations guaranteeing closed loop stability. Such a terminal set is ignored in eq. (9) for reasons of notational simplicity, but it adding such a terminal set would be straight forward.

From eq. (9), it is clear that $\delta_{i}^{z}$ and $\delta_{i}^{v}$ have to be found in order to be able to formulate the nominal MPC. This will be addressed in Section 4.

\section{Digital Sliding Mode Control}

To design the auxiliary digital SMC, we consider the (9) deviated system dynamics described by eq. (8). Two

\footnotetext{
${ }^{1}$ The cost function will not be specified at present, but we do naturally assume a sensible cost function ensuring that the control of the nominal system is stable, and one which allows the optimization problem to be solved efficiently.
} 
control algorithms are implemented herein. The first one is a traditional relay based sliding mode control defined by

$$
\nu_{k}=-(K B)^{-1}\left(K A \epsilon_{k}-g_{k}+\Delta_{u} \operatorname{sign}\left(g_{k}\right)\right)
$$

where

$$
g_{k}=K \epsilon_{k}
$$

denotes switching function and

$$
g_{k}=0
$$

is the equation for the sliding surface or the intersection of sliding surfaces if $n_{u}>1$. Notice that $K \epsilon_{k}$ is usually selected as an auxiliary control in Tube MPC and a matrix $K$ has dimension $n_{u} \times n_{x}$. Here $\operatorname{sign}\left(g_{k}\right)$ is understood to be a vector with elements \pm 1 , and $\Delta_{u}$ is a diagonal matrix with constants representing the relay outputs. The sliding surface, i.e. $K$, should be selected so that the system (Furuta, 1990)

$$
\begin{aligned}
\epsilon_{k+1} & =\left(A-B(K B)^{-1} K(A-I)\right) \epsilon_{k} \\
K \epsilon_{k} & =0
\end{aligned}
$$

is stable. Eqs. (13) and (14), describing system dynamics in sliding mode, are obtained by implementing the well-known equivalent control

$$
\nu_{k}^{e q c}=-(K B)^{-1}\left(K A \epsilon_{k}-g_{k}\right)
$$

in eq. (8). Substituting eq. (10) in eqs. (8) and (11) yields

$$
g_{k+1}=g_{k}-\Delta_{u} \operatorname{sign}\left(g_{k}\right)+K E w_{k}
$$

defining the switching function dynamics at time instant $k$, whereas in the prediction horizon it is determined by

$$
\begin{aligned}
g_{k+i+1} & =g_{k+i}-\Delta_{u} \operatorname{sign}\left(g_{k+i}\right)+K E w_{k+i}, \\
i & \in\{0,1, \ldots, N\}
\end{aligned}
$$

In order to provide stable switching function dynamics, $\Delta_{u}$ should be calculated according to the following theorem.

Theorem 3.1. If $\Delta_{u}$ is chosen to satisfy the following inequality

$$
\Delta_{u} \underline{1}>\Omega>\max \left|K E w_{k}\right|,
$$

where $\Omega$ is a positive real vector and 1 is a vector of 1 's, then, for every initial state $g_{k}$, there exists a positive integer number $k_{0}=k_{0}\left(g_{k}\right)<N$, such that the system phase trajectory, described by eqs. (17) and (18), enters the domain defined by

$$
G=\left\{g_{k+i}:\left|g_{k+i}\right|<\Delta_{u} \underline{1}+\Omega\right\},
$$

after $k_{0}$ time steps and remains in this domain for all $i>k_{0}$.
Proof. See Appendix A.

The second auxiliary digital SMC, used in this paper, is so-called robust discrete-time chattering free sliding mode control (Golo and Milosavljević, 2000)

$$
\begin{aligned}
\nu_{k}= & -(K B)^{-1}\left(K A \epsilon_{k}-g_{k}\right. \\
& \left.+\min \left(I\left|g_{k}\right|, \Delta_{u}\right) \operatorname{sign}\left(g_{k}\right)\right)
\end{aligned}
$$

Implementing eq. (20) in eq. (8), the switching function dynamics becomes

$$
g_{k+1}=g_{k}-\min \left(I\left|g_{k}\right|, \Delta_{u}\right) \operatorname{sign}\left(g_{k}\right)+K E w_{k}
$$

and, inside the prediction horizon, we have

$$
\begin{aligned}
g_{k+i+1} & =g_{k+i}-\min \left(I\left|g_{k+i}\right|, \Delta_{u}\right) \operatorname{sign}\left(g_{k+i}\right) \\
& +K E w_{k+i}, \quad i \in\{0,1, \ldots, N\}
\end{aligned}
$$

The next theorem gives sufficient conditions for stable sliding motion in prediction horizon.

Theorem 3.2. The system phase trajectory, described by eqs. (22) and (18), reaches the domain $G$ defined by eq. (19) in $k_{0}=k_{0}\left(g_{k}\right)<N$ time steps for every initial $g_{k}$, and remains in it for all $i>k_{0}$.

Proof. See Appendix B.

\section{Calculating the required constraint tightening with SMC-based auxiliary controllers}

We will first describe how to calculate the required constraint tightening with control defined by eq. (10). However, relay feedback is known to often result in very fast switching, which for some applications will not be desirable. A common remedy is then to replace the 'infinite gain' at the switching surface with a steep linear function, leading to a chattering free SMC described by eq. (20). The second subsection will address the constraint tightening for this type of auxiliary controller.

It is noted above that an auxiliary controller with low calculation requirements may operate at a higher sampling rate (shorter timestep) than the MPC. However, we will use the same sampling frequency both for MPC and SMC.

With the simple SMC-inspired auxiliary controllers considered here, the determination of $\delta_{i}^{v}$ is trivial, which will become apparent below. However, the calculation of $\delta_{i}^{z}$ is more challenging. 


\subsection{Constraint tightening for traditional SMC}

Denote the relay term in eq. (10) as

$$
\vartheta_{k}=\Delta_{u} \operatorname{sign}\left(K \epsilon_{k}\right),
$$

To proceed, we define for each element $\vartheta_{j}$ of $\vartheta$ a binary variable $s_{j}$, such that $\vartheta_{j}>0 \Rightarrow s_{j}=1$, and $s_{j}=0$ otherwise. Also needed are upper and lower bounds on each component of the vector $K \epsilon$. From eqs. (2) and (5), it is clearly safe to assume $F \epsilon \leq f$, and the lower bounds $m_{j}$ and upper bounds $\mu_{j}$ on element $j$ of $K \epsilon$ can be found from the LPs

$$
m_{j}=\min _{F \epsilon \leq f} K_{j} \epsilon
$$

and

$$
\mu_{j}=\max _{F \epsilon \leq f} K_{j} \epsilon
$$

where $K_{j}$ is row $j$ of $K$. Let $\underline{1}$ denote a vector of 1 's. Equation (23) is then implied by Mignone (2001)

$$
\vartheta_{k}=\Delta_{u} S-\Delta_{u}(\underline{1}-s)=\Delta_{u}(2 s-\underline{1}),
$$

where the value of the binary variables $s$ follow from the constraints

$$
\begin{aligned}
m_{j}\left(1-s_{j}\right) & <K_{j} \epsilon \\
-\mu_{j} s_{j} & <-K_{j} \epsilon
\end{aligned}
$$

Note that numerical optimization solvers cannot distinguish between strict and non-strict inequalities. The formulation above will leave the value of $s_{j}$ undecided if $K_{j} \epsilon=0$. It will then be left for the optimization routine to choose the optimal value.

The furthest from the origin the disturbance sequence $\left\{w_{k}\right\}$ may drive the deviation state $\epsilon_{k}$ in the direction of the state constraint $F_{l} x_{k} \leq f_{l}$ over a horizon of $N$ timesteps can then be found by solving

$$
\delta f_{j, N}=\max _{w_{k}, s_{k}, \epsilon_{k}} F_{l} \epsilon_{N}
$$

subject to

$$
\begin{gathered}
\epsilon_{0}=0 \\
H w_{k} \leq h ; k=0, \ldots, N-1 \\
\epsilon_{k+1}=\bar{A} \epsilon_{k}+\bar{B} \Delta_{u}\left(2 s_{k}-\underline{1}\right)+E w_{k} ; \\
k=0, \ldots, N-1 \\
\bar{A}=A-B(K B)^{-1} K(A-I) \\
\bar{B}=-B(K B)^{-1} \\
\operatorname{diag}\left\{m_{j}\right\}\left(\underline{1}-s_{k}\right)<K \epsilon_{k} ; k=0, \ldots, N-1 \\
-\operatorname{diag}\left\{\mu_{j}\right\} s_{k}<-K \epsilon_{k} ; k=0, \ldots, N-1 \\
s_{k} \in\{0,1\}^{n_{u}}
\end{gathered}
$$

For each state constraint $j$, this optimization should be solved for a number of horizon lengths $N$. For each timestep $i$, the elements of $\delta_{i}^{z}$ are given by $\delta f_{j, i}$. If the system under the relay feedback is stable, $\delta f_{j, N}$ will approach an upper bound as $N$ grows large.

\subsection{Constraint tightening for chattering free SMC}

Instead of eq. (23) we now use

$$
\vartheta_{j}=\min \left(\left|K_{j} \epsilon\right|, \Delta_{u j}\right) \operatorname{sign}\left(K_{j} \epsilon\right)
$$

where $\Delta_{u j}$ denotes the $j$ 'th element on the main diagonal of the diagonal matrix $\Delta_{u}$, and $K_{j}$ as before refers to row $j$ of $K$. We rewrite eq. (30) as

$$
\vartheta_{j}=\operatorname{sat}\left(K_{j} x\right)=\left\{\begin{aligned}
\Delta_{u j} & \text { if } K_{j} \epsilon \geq \Delta_{u j} \\
K_{j} \epsilon & \text { if }-\Delta_{u j} \leq K_{j} \epsilon \leq \Delta_{u j} \\
-\Delta_{u j} & \text { if } K_{j} \epsilon \leq-\Delta_{u j}
\end{aligned}\right.
$$

To capture this behavior, we need two binary variables, $s_{j}$ and $t_{j}$ for each auxiliary input $\vartheta_{j}$, such that

$$
\begin{array}{ccc}
K_{j} \epsilon & <-\Delta_{u j} & \rightarrow s_{j}=0 \\
K_{j} \epsilon & >-\Delta_{u j} & \rightarrow s_{j}=1 \\
K_{j} \epsilon & <\Delta_{u j} & \rightarrow t_{j}=0 \\
K_{j} \epsilon & >\Delta_{u j} & \rightarrow t_{j}=1
\end{array}
$$

Define

$$
q_{j}=K_{j} \epsilon-m_{j}
$$

where $m_{j}$ is calculated as in eq. (24). We note that $q_{j}$ is non-negative in the domain of interest. The actual input from the auxiliary controller may then be calculated from the expression

$$
\vartheta_{j}=-\left(1-s_{j}\right) \Delta_{u j}+\left(s_{j}-t_{j}\right)\left(q_{j}+m_{j}\right)+t_{j} \Delta_{u j}
$$

where we note that $s_{j} \geq t_{j}$. The difficulty in the above equation lies in the bilinear terms $s_{j} q_{j}$ and $t_{j} q_{j}$, both being the product of a binary variable and a nonnegative real. To proceed, we introduce the auxiliary variables $\sigma_{j}=s_{j} q_{j}$ and $\tau_{j}=t_{j} q_{j}$. From (Bemporad and Morari, 1999), we have that the set

$\mathcal{R}=\left\{\left(q_{j}, s_{j}, \sigma_{j}\right): \sigma_{j}=s_{j} q_{j}, 0 \leq q_{j} \leq a_{j}, s_{j} \in\{0,1\}\right\}$

can equivalently be expressed as

$$
\mathcal{M}=\begin{gathered}
\left\{\left(q_{j}, s_{j}, \sigma_{j}\right): 0 \leq \sigma_{j} \leq a_{j} s_{j},\right. \\
\left.q_{j}+a_{j} s_{j}-a_{j} \leq \sigma_{j} \leq q_{j}, s_{j} \in\{0,1\}\right\}
\end{gathered}
$$

and similarly for $\left(q_{j}, t_{j}, \tau_{j}\right)$. Recognizing that in this case $a_{j}=\mu_{j}-m_{j}$, and introducing

$$
\begin{aligned}
m_{1 j} & =m_{j}+\Delta_{u j} \\
\mu_{1 j} & =\mu_{j}+\Delta_{u j} \\
m_{2 j} & =m_{j}-\Delta_{u j} \\
\mu_{2 j} & =\mu_{j}-\Delta_{u j}
\end{aligned}
$$


Defining the diagonal matrices $\Lambda=\operatorname{diag}\left(a_{j}\right), \underline{M}_{1}=$ $\operatorname{diag}\left(m_{1 j}\right), \bar{M}_{1}=\operatorname{diag}\left(\mu_{1 j}\right), \underline{M}_{2}=\operatorname{diag}\left(m_{2 j}\right)$, and $\bar{M}_{2}=\operatorname{diag}\left(\mu_{2 j}\right)$, and forming the column vectors $m=$ $\operatorname{vec}\left(m_{j}\right), s_{k}=\operatorname{vec}\left(s_{k j}\right), t_{k}=\operatorname{vec}\left(t_{k j}\right), \sigma_{k}=\operatorname{vec}\left(\sigma_{k j}\right)$, and $\tau_{k}=\operatorname{vec}\left(\tau_{k j}\right)$, we obtain the optimization formulation

$$
\begin{aligned}
& \delta f_{l, N}=\max _{w_{k}, \vartheta_{k}, \sigma_{k}, \tau_{k}, s_{k}, t_{k}, \epsilon_{k}} F_{l} \epsilon_{N} \\
& \text { subject to } \\
& \epsilon_{0}=0 \\
& H w_{k} \leq h ; k=0, \ldots, N-1 \\
& \epsilon_{k+1}=\bar{A} \epsilon_{k}+\bar{B} \vartheta_{k}+E w_{k} ; k=0, \ldots, N-1 \\
& \vartheta_{k}=-\Delta_{u}\left(\underline{1}-s_{k}\right)+M\left(s_{k}-t_{k}\right)+\sigma_{k}-\tau_{k} \\
& +\Delta_{u} t_{k} ; k=0, \ldots, N-1 \\
& \underline{M}_{1}\left(\underline{1}-s_{k}\right)<K \epsilon_{k}+\Delta_{u} \underline{1} ; \quad k=0, \ldots, N-1 \\
& -\bar{M}_{1} s_{k}<-K \epsilon_{k}-\Delta_{u} \underline{1} ; k=0, \ldots, N-1 \\
& \underline{M}_{2}\left(\underline{1}-t_{k}\right)<K \epsilon_{k}-\Delta_{u} \underline{1} ; \quad k=0, \ldots, N-1 \\
& -\bar{M}_{2} t_{k}<-K \epsilon_{k}+\Delta_{u} \underline{1} ; \quad k=0, \ldots, N-1 \\
& \sigma_{k}>0 ; k=0, \ldots, N-1 \\
& \tau_{k}>0 ; k=0, \ldots, N-1 \\
& \sigma_{1 k}<\Lambda s_{k} ; k=0, \ldots, N-1 \\
& \tau_{k}<\Lambda t_{k} ; k=0, \ldots, N-1 \\
& \sigma_{k}<K \epsilon_{k}-m ; k=0, \ldots, N-1 \\
& \tau_{k}<K x_{k}-m ; k=0, \ldots, N-1 \\
& K \epsilon_{k}+\Lambda s_{k}-\Lambda \underline{1}-m<\sigma_{k} ; k=0, \ldots, N-1 \\
& K \epsilon_{k}+\Lambda t_{k}-\Lambda \underline{1}-m<\tau_{k} ; k=0, \ldots, N-1 \\
& s_{k} \in\{0,1\}^{n_{u}}, \quad t_{k} \in\{0,1\}^{n_{u}}
\end{aligned}
$$

Clearly, with $\nu_{k}$ as in eqs. (10) and (20) taking into account $F \epsilon \leq f$ as specified above, we have

$$
\delta_{i}^{v}=\max \left(\nu_{k}\right) \forall i .
$$

\section{Digital simulation and experimental results}

The validation of the proposed control methods is performed by using the modular servo system (Inteco, 2011) shown in Figure 2. The objective is to control the angular position of the DC motor shaft. The system consists of the following components: a tachogenerator, a DC motor, an encoder and an inertia load. This modular experimental setup supports real-time design and implementation of advanced control algorithms, and is interfaced with the MATLAB/Simulink using specific RT-DAC4/USB board for transferring the measured signals from the tachogenerator and encoder, and the control signals to the power interface unit. The angular position $\theta$ of the DC motor shaft is measured by the incremental encoder, and the angular velocity $\omega$ is

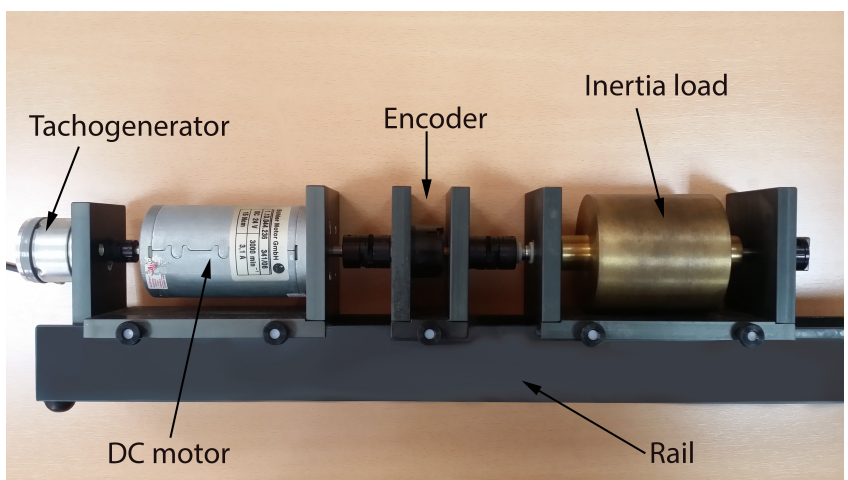

Figure 2: DC servo system setup

proportional to the voltage produced by the tachogenerator. The DC motor is controlled by a PWM signal with the scaled input voltage

$$
U(t)=V(t) / V_{\max }
$$

where $|U(t)| \leq 1$ and $V_{\max }=12[V]$.

In order to identify the model of the system, the identification tool within Modular Servo Toolbox, which operates directly in the MATLAB/Simulink environment, is used. The identification procedure is also given in (Inteco, 2011). The following transfer function is obtained

$$
G(s)=\frac{\theta(s)}{u(s)}=\frac{K_{s}}{s\left(T_{s} s+1\right)}
$$

where $K_{s}=184.73$ and $T_{s}=1.3 s$.

By denoting $x_{1}=\theta$ and $x_{2}=\omega$, the state space model of servo system is

$$
\begin{aligned}
& \dot{x}_{1}=x_{2} \\
& \dot{x}_{2}=a x_{2}+b u+w
\end{aligned}
$$

where $a=-1 / T_{s}$ and $b=K_{s} / T_{s}$, and $w$ represents the Coulomb friction defined by

$$
w=F_{c} \operatorname{sign}\left(x_{2}\right)
$$

treated as the unmodeled disturbance.

The sampling period is set to $T=0.01 \mathrm{~s}$, and the discrete-time state space model is given by

$$
\begin{aligned}
x_{k+1} & =A x_{k}+B u_{k}+E w_{k} \\
y_{k} & =C x_{k}
\end{aligned}
$$

with

$$
\begin{aligned}
& A=\left[\begin{array}{cc}
1 & 0.01 \\
0 & 0.9923
\end{array}\right] \\
& B=\left[\begin{array}{l}
0.0071 \\
1.4155
\end{array}\right] \\
& E=\left[\begin{array}{l}
0 \\
1
\end{array}\right] \\
& C=\left[\begin{array}{ll}
1 & 0
\end{array}\right] .
\end{aligned}
$$


The weight matrices are chosen as

$$
\begin{array}{r}
Q=\left[\begin{array}{cc}
50 & 0 \\
0 & 1
\end{array}\right] \\
R=1000
\end{array}
$$

and the prediction horizon of $N=20$ is considered.

The dynamics described by eq. (68) is split into the nominal one, eq. (7), and the deviation from the nominal, eq. (8).

Three sets of the digital simulations and real-time experiments are conducted in order to validate the proposed Tube MPC control methods. The reference signal is defined by

$$
r=\left\{\begin{aligned}
0 & \text { if Time steps }<50 \\
40 & \text { if } 50 \leq \text { Time steps } \leq 500 \\
0 & \text { if Time steps }>500
\end{aligned}\right.
$$

In all three sets, the nominal MPC, $v$, is calculated by the nominal model only, and SMC, $\nu$, is used as the auxiliary controller to eliminate the disturbance.

\section{A. Nominal $M P C$}

In order to show the system response, when only the nominal MPC is applied, the first set of the digital simulation and real-time experiment is conducted. The following control

$$
-1 \leq u \leq 1
$$

and the state

$$
\begin{aligned}
& -50 \leq x_{1} \leq 50 \\
& -34 \leq x_{2} \leq 34
\end{aligned}
$$

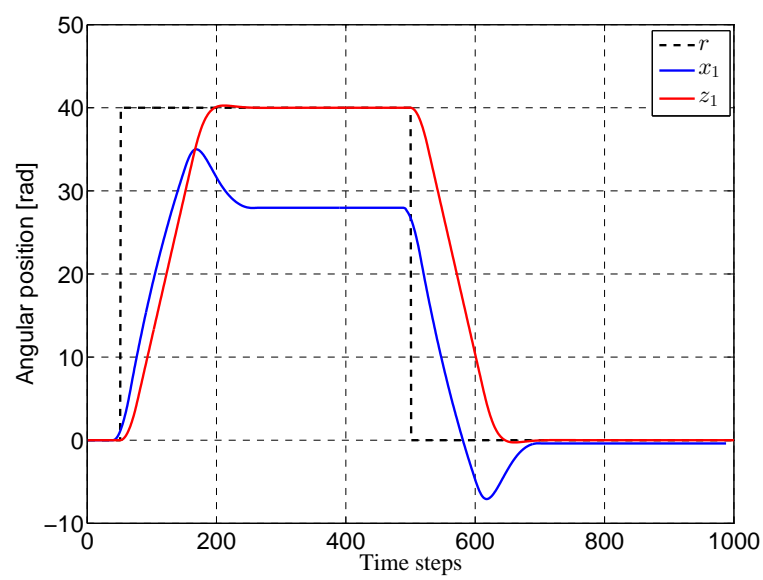

Figure 3: The angular position $z_{1}$ of the nominal model, and $x_{1}$ of the real plant for the nominal MPC

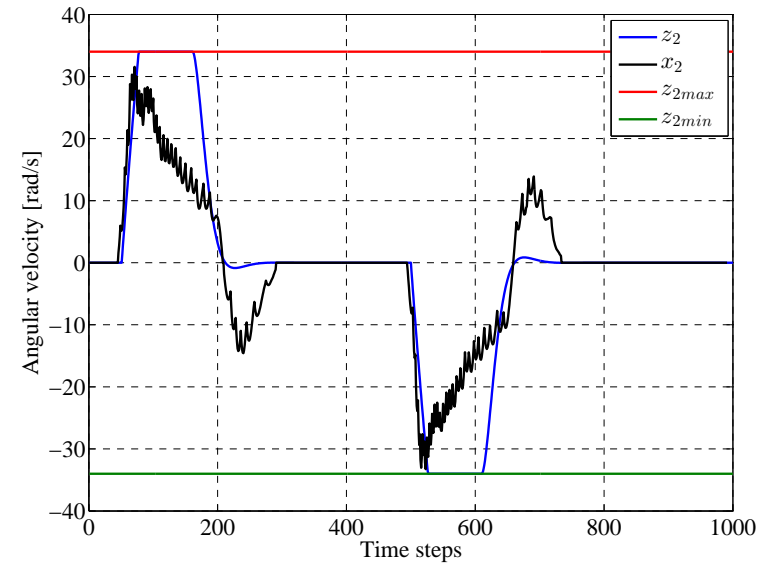

Figure 4: The angular velocity $z_{2}$ of the nominal model, and $x_{2}$ of the real plant for the nominal MPC

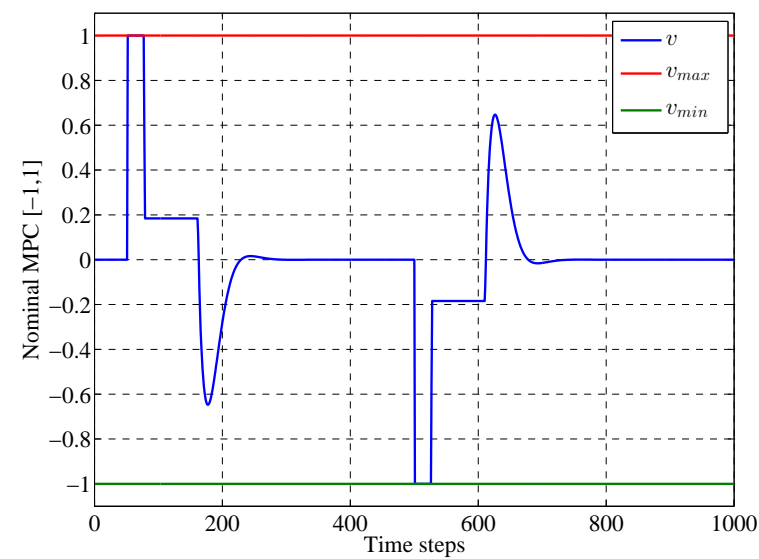

Figure 5: Nominal MPC signal

constraints are defined.

Initially, the nominal MPC, shown in Figure 5, is applied to the nominal model. The digital simulation results, together with the corresponding experimental results, are depicted in Figures 3 and 4.

It is shown that both nominal and real states respect the constraints defined by eq. (74), but there is discrepancy between the responses of the nominal model and real plant. This demonstrates the lack of robustness of the nominal MPC when it is applied to the real-time DC servo system in the presence of disturbance.

\section{B. Tube MPC with traditional SMC}

The traditional SMC defined by eq. (10), as an auxiliary controller of Tube MPC, is applied to cope with the disturbance. The two control components are now 
constrained separately.

The constraints for the nominal MPC and SMC are defined by

$$
\begin{aligned}
& -0.7 \leq v_{k} \leq 0.7 \\
& -0.3 \leq \nu_{k} \leq 0.3
\end{aligned}
$$

which satisfy eq. (73), i.e. $-1 \leq v+\nu \leq 1$. The new state constraints are calculated by using the tightening procedure described in Section 4. The tightened state constraints used for the nominal system are now defined by

$$
\begin{aligned}
& -45 \leq z_{1} \leq 45 \\
& -25 \leq z_{2} \leq 25
\end{aligned}
$$

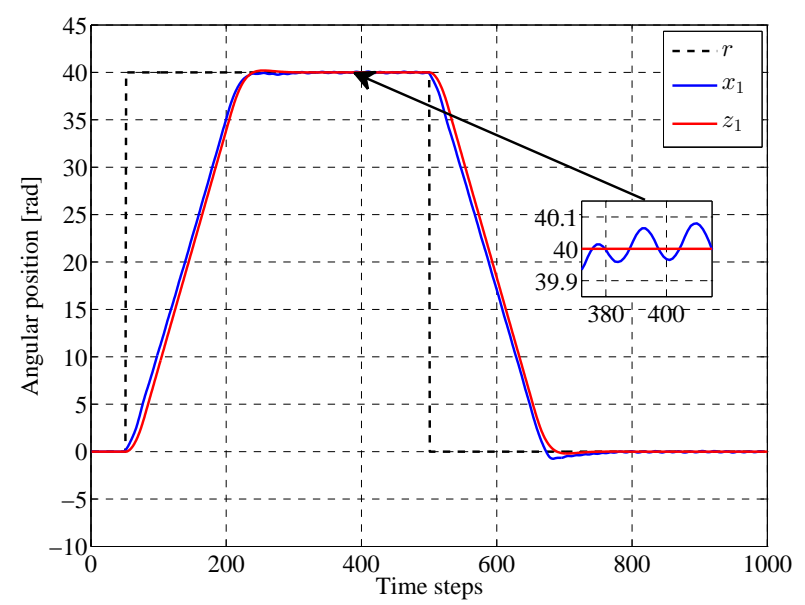

Figure 6 : The angular position $z_{1}$ of the nominal model for the nominal MPC, and $x_{1}$ of the real plant for the proposed control

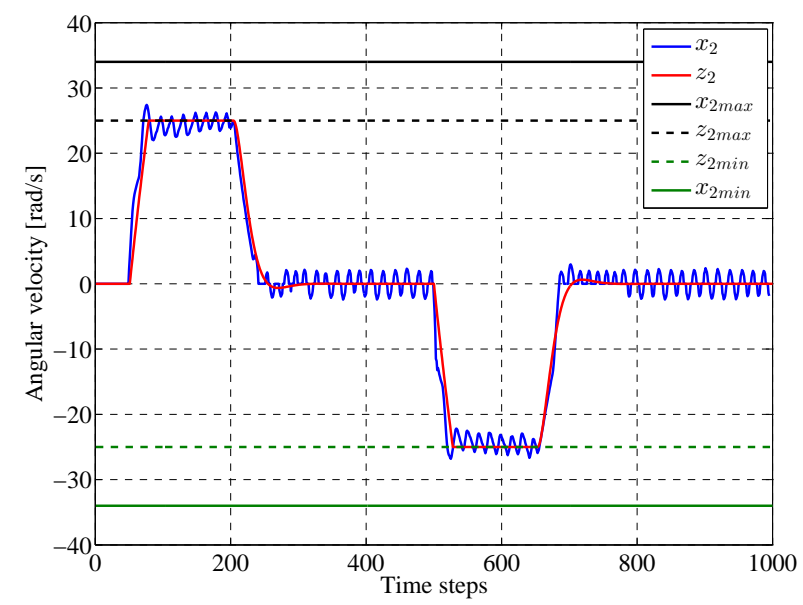

Figure 7: The angular velocity $z_{2}$ of the nominal model for the nominal MPC, and $x_{2}$ of the real plant for the proposed control and the real system has to satisfy constraints defined by eq. (74). First, the digital simulation is performed, where nominal MPC signal is applied to the nominal model. Obtained results are shown in Figures 6 and 7. It can be seen that the nominal states respect constraints defined by eq. (77). The nominal MPC signal also respects the constraints defined by eq. (75), which is illustrated in Figure 8. Then, the Tube MPC with the traditional auxiliary SMC is applied to the real-time DC servo system in order to eliminate the disturbance. The parameters of the SMC component are $\Delta_{u}=0.3$ and $K=[-0.0118-0.0071]$. The real-time system responses are also presented in Figures 6 and 7. In Figure 9 is presented the SMC component of the Tube MPC. Comparing the previous two experimental results, it is shown that the disturbance is rejected, but there is a little chattering in the

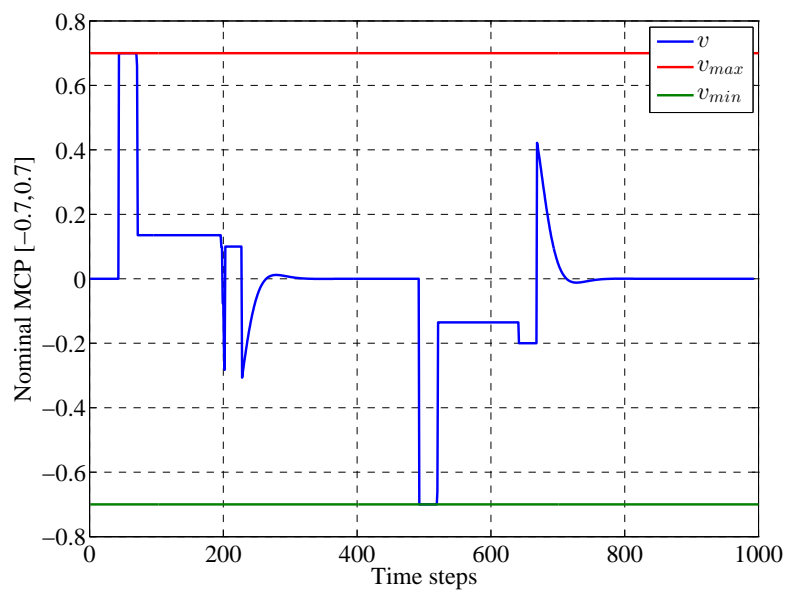

Figure 8: Nominal MPC component of the proposed control

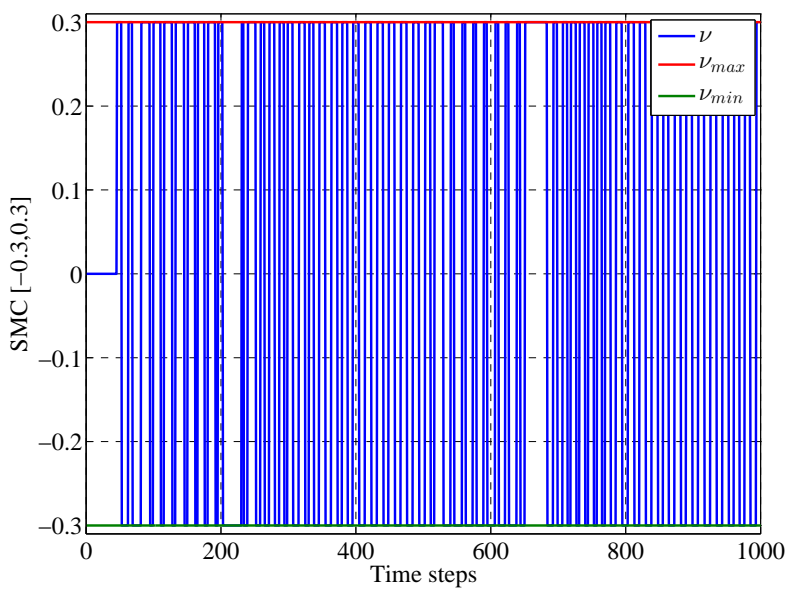

Figure 9: Traditional SMC component of the proposed control 
output signal. The next experiment demonstrates how to eliminate the chattering phenomenon.

\section{Tube MPC with chattering free $S M C$}

The same control and state constraints, defined by eqs. (75), (76) and (77), respectively, are used herein. The nominal MPC is applied to the nominal model first. The obtained nominal and real-time system responses are illustrated in Figures 10 and 11. After that, the Tube based MPC with the chattering free $\mathrm{SMC}$ is applied to the real DC servo system. The SMC component is defined by eq. (20) and the parameters are $\Delta_{u}=0.3$ and $K=[-0.0118-0.0071]$. Figure 10 shows that the chattering is eliminated. The oscillations in SMC component between 0 and 200, as well as 500 and 700 time steps in Figure 13 origi-

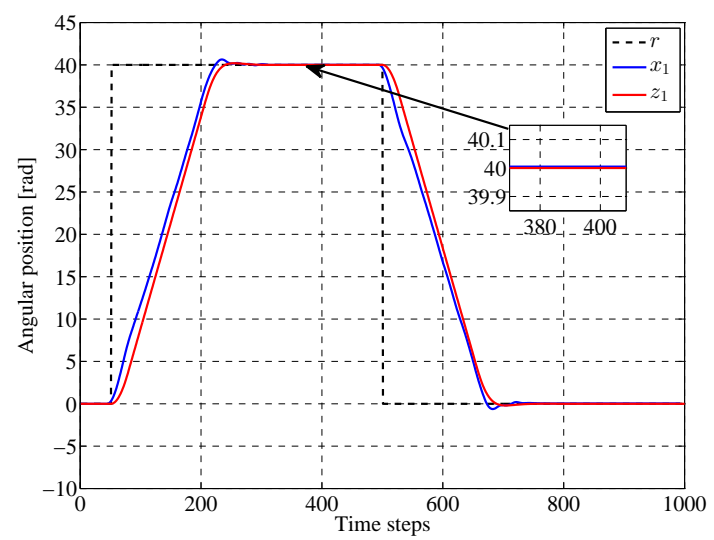

Figure 10: The angular position $z_{1}$ of the nominal model for the nominal MPC, and $x_{1}$ of the real plant for the proposed control

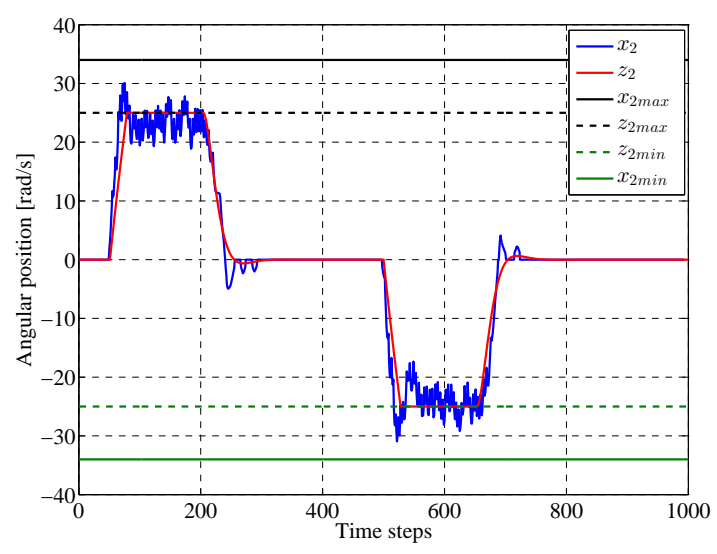

Figure 11: The angular velocity $z_{2}$ of the nominal model for the nominal MPC, and $x_{2}$ of the real plant for the proposed control

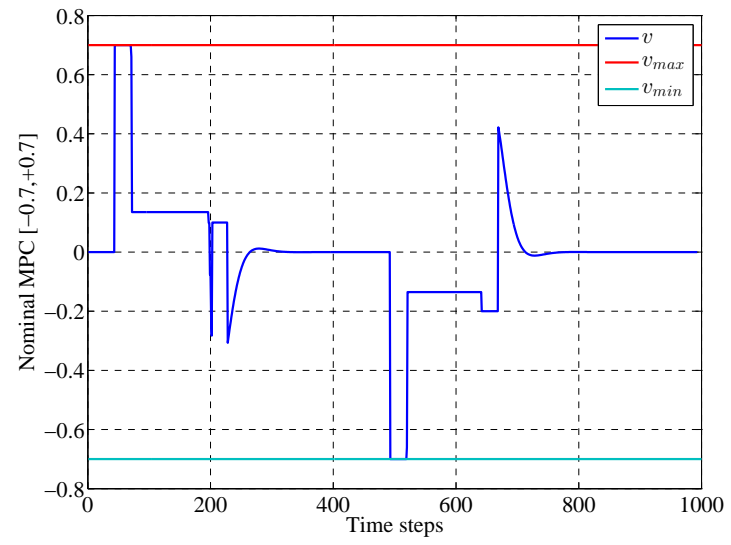

Figure 12: Nominal MPC component of the proposed control

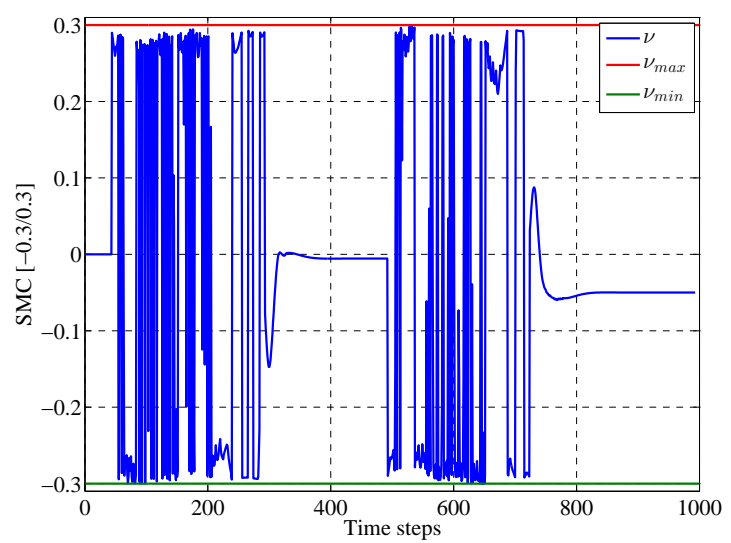

Figure 13: Chattering free SMC component of the proposed control

nate from noise existing in angular velocity signal taken from tachogenerator (Figure 11 ). Therefore, they are not caused by chattering phenomenon. As in the previous experiments, all states and control signals respect the defined constraints.

\section{Conclusion}

In this paper, the Tube MPC with a SMC as an auxiliary controller is studied in order to improve the robustness of the overall system. Due to the presence of the SMC component, it is necessary to tighten the constraints of the nominal MPC part. The online calculations for SMC are not time consuming and this is also true for the nominal MPC, handling only the nominal model, which results in lower online calculation requirements. The traditional and chattering-free SMC algorithms are introduced in Tube MPC in order to reject disturbances and to achieve better perfor- 
mances of the real system. Therefore, the procedures for calculating the required constraints tightening are derived for the both cases. The good characteristics of the proposed control algorithms are demonstrated by conducting several digital simulations and real-time experiments on the DC servo system.

\section{Acknowledgments}

This work is supported by Norwegian Ministry of Foreign Affairs, grant 2011/1383, under Programme for Higher Education, Research and Development (HERD), project NORBAS, that is leaded by Norwegian University of Science and Technology.

\section{Appendix A}

\section{Proof of Theorem 3.1}

The vector sequence $\left(g_{k}, g_{k+1}, \ldots, g_{k+i}, \ldots\right)$, denoted by $\left(g_{k+i}\right)$, converges point-wise to the limit $g \in \mathbb{R}^{n_{u}}$ if each element of $g_{k+i}$ converges to the corresponding element in $g$. In other words, $\left(g_{k+i}\right)$ is convergent if $\lim g_{k+i}=g$, i.e. if for every real vector $\epsilon>\mathbf{0}$ there exists the natural number $N_{u}(\epsilon)$, such that

$$
\left|g_{k+i}-g\right|<\epsilon, \quad \forall i>N_{u}(\epsilon)
$$

$\left(g_{k+i}\right)$ is the positive (negative) vector sequence if $g_{k+i} \geq \mathbf{0}\left(g_{k+i} \leq \mathbf{0}\right)$ for $i=0,1,2, \ldots$ For multipleinput systems, it is probable that the elements of vector $g_{k}$ have different signs, as they represent the switching functions of SMC inputs. After splitting the vector $g_{k}$ onto two sub vectors $g_{k}^{+}$and $g_{k}^{-}$with separated positive and negative elements of $g_{k}$, respectively, eq. (17) can be rewritten as

$$
\begin{aligned}
& g_{k+i+1}^{+}=g_{k+i}^{+}-\Delta_{u}^{+} \operatorname{sign}\left(g_{k+i}^{+}\right)+(K E)^{+} w_{k+i}^{+} \\
& g_{k+i+1}^{-}=g_{k+i}^{-}-\Delta_{u}^{-} \operatorname{sign}\left(g_{k+i}^{-}\right)+(K E)^{-} w_{k+i}^{-}
\end{aligned}
$$

where $\Delta_{u}^{+}, \Delta_{u}^{-},(K E)^{+},(K E)^{-}, w_{k+i}^{+}$and $w_{k+i}^{-}$are diagonal matrices and sub vectors obtained from $\Delta_{u}$, $K E$ and $w_{k+i}$ by extraction. Similarly, the theorem's condition given in eq. (18) can be expressed by

$$
\begin{aligned}
& \Delta_{u}^{+} \underline{1}>\Omega^{+}>\max \left|(K E)^{+} w_{k}^{+}\right| \\
& \Delta_{u}^{-} \underline{1}>\Omega^{-}>\max \left|(K E)^{-} w_{k}^{-}\right|
\end{aligned}
$$

and the domain $G$ defined by eq. (19) as

$$
G=G^{+} \cup G^{-}
$$

with

$$
\begin{aligned}
& G^{+}=\left\{g_{k+i}^{+}:\left|g_{k+i}^{+}\right|<\Delta_{u}^{+} \underline{1}+\Omega^{+}\right\} \\
& G^{-}=\left\{g_{k+i}^{-}:\left|g_{k+i}^{-}\right|<\Delta_{u}^{-} \underline{1}+\Omega^{-}\right\}
\end{aligned}
$$

It is obvious that $\left(g_{k+i}^{+}\right)$and $\left(g_{k+i}^{-}\right)$, defined by eq. (A.2), are positive and negative sequences, respectively.

Let us prove now that $\left(g_{k+i}^{+}\right)$enters the domain $G^{+}$ in finite time for $k_{0} \leq i \leq N$ and remains in that area. The proof is similar in the case of $\left(g_{k+i}^{-}\right)$with respect to $G^{-}$. If eq. (A.4) is true, then

$$
\begin{aligned}
g_{k+i+1}^{+}-g_{k+i}^{+} & =-\Delta_{u}^{+} \operatorname{sign}\left(g_{k+i}^{+}\right)+(K E)^{+} w_{k+i}^{+} \\
& <-\Delta_{u}^{+} \underline{1}+\Omega^{+}<\mathbf{0}
\end{aligned}
$$

and $g_{k+i+1}^{+}<g_{k+i}^{+}$so there exists a positive diagonal matrix $Q_{k+i}=\operatorname{diag}\left\{\begin{array}{llll}q_{k+i}^{1} & q_{k+i}^{2} & \ldots & q_{k+i}^{n_{u}^{+}}\end{array}\right\}$, $\left(0<q_{k+i}^{j}<1, j=1,2, \ldots, n_{u}^{+}, n_{u}^{+}+n_{u}^{-}=n_{u}\right)$ such that

$$
g_{k+i+1}^{+}=Q_{k+i} g_{k+i}^{+}, \quad Q_{k+i}<I
$$

where $g_{k+i}^{+}$and $g_{k+i+p}^{-}(p \in N)$ can be written as

$$
\begin{aligned}
g_{k+i}^{+} & =\left(\prod_{j=k}^{k+i-1} Q_{j}\right) g_{k}^{+} \\
g_{k+i+p}^{+} & =\left(\prod_{j=k}^{k+i+p-1} Q_{j}\right) g_{k}^{+}
\end{aligned}
$$

giving the following inequality $(\epsilon>\mathbf{0})$

$$
\begin{aligned}
& \left|g_{k+i+p}^{+}-g_{k+i}^{+}\right| \\
& =\left|\left(\prod_{j=k}^{k+i-1} Q_{j}\right)\left(\left(\prod_{l=k+i}^{k+i+p-1} Q_{l}\right)-I\right) g_{k}^{+}\right|<\epsilon
\end{aligned}
$$

According to Cauchy's theorem, the convergence of vector sequence $\left(g_{k+i}\right)$, satisfying eq. (A.13), is proved. Its convergence domain is

$$
\bar{G}^{+}=\left\{g_{k+i}^{+}:\left|g_{k+i}^{+}\right|>\Delta_{u}^{+} \underline{1}+\Omega^{+}\right\}
$$

directly satisfying eq. (A.10).

Let us now show that system trajectory enters the domain $G^{+}$in finite time. The sequence $\left(g_{k+i}^{+}\right)$ converges inside domain $\bar{G}^{+}$, so it is limited and $\lim _{i \rightarrow \infty} g_{k+i}^{+}=g_{\infty}^{+}$. Assume that $g_{k}^{+}>\Delta_{u}^{+} \underline{1}+\Omega^{+}$. According to eq. (A.2)

$$
g_{k+i}^{+}=g_{k}^{+}-\sum_{j=0}^{k+i-1}\left(\Delta_{u}^{+} \underline{1}-(K E)^{+} w_{k+j}^{+}\right) .
$$

Suppose that $g_{k+i}^{+}$never enters the domain $G^{+}$. For $i \rightarrow \infty$, we obtain

$$
\sum_{j=0}^{\infty}\left(\Delta_{u}^{+} \underline{1}-(K E)^{+} w_{k+j}^{+}\right)<g_{k}^{+}-\Delta_{u}^{+} \underline{1}-\Omega^{+} .
$$


Equation (A.16) implies that the vector series

$$
\sum_{j=0}^{\infty}\left(\Delta_{u}^{+} \underline{1}-(K E)^{+} w_{k+j}^{+}\right)
$$

is convergent, and its general element $\Delta_{u}^{+} \underline{1}-$ $(K E)^{+} w_{k+j}^{+}$converges to zero as $j \rightarrow \infty$, i.e.

$$
\Delta_{u}^{+} \underline{1}=\lim _{j \rightarrow \infty}\left((K E)^{+} w_{k+j}^{+}\right)
$$

that contradicts eq. (A.4), and the initial assumption that $g_{k+i}^{+}$never enters the domain $G^{+}$is false. Moreover, $g_{k+i}^{+}$enters the domain $G^{+}$at time instant $k_{0}$ which is bounded by the maximal element of vector

$$
\overline{k_{0}}=\operatorname{int}\left(\left(\left(\Delta_{u}^{+} \underline{1}-\Omega^{+}\right) I\right)^{-1}\left(\left|g_{k}^{+}\right|-\Delta_{u}^{+} \underline{1}-\Omega^{+}\right)\right)+\underline{1}
$$

It is obvious that the length of the prediction horizon $N$ should be greater than $k_{0}$ and selected in accordance with eq. (A.18).

We will now show that for every $k_{0}<i<N, g_{k+i}^{+}$ remains in the domain $G^{+}$. Let $s_{k+k_{0}} \in G_{+}^{+}=\left\{g_{k+i}^{+}\right.$: $\left.0<g_{k+i}^{+}<\Delta_{u}^{+} \underline{1}+\Omega^{+}\right\}$. Then, according to eq. (A.2), we have

$$
\begin{aligned}
& -\Delta_{u}^{+} \underline{1}-\Omega^{+} \underset{(A .4)}{<}-\Delta_{u}^{+} \underline{1}+(K E)^{+} w_{k+k_{0}}^{+} \\
& \underset{(A .4)}{<} g_{k+k_{0}}^{+}-\Delta_{u}^{+} \underline{1}+(K E)^{+} w_{k+k_{0}}^{+} \\
& =g_{k+k_{0}+1}^{+} \underset{(A .4)}{<} 2 \Omega^{+}<\Delta_{u}^{+} \underline{1}+\Omega^{+}
\end{aligned}
$$

and thus $g_{k+i}^{+}$does not leave the domain $G^{+}$. This is also true when $g_{k+k_{0}}^{+} \in G_{-}^{+}=\left\{g_{k+i}^{+}:-\Delta_{u}^{+} \underline{1}-\Omega^{+}<\right.$ $\left.g_{k+i}^{+}<0\right\}$ since

$$
\begin{aligned}
& -\Delta_{u}^{+} \underline{1}-\Omega^{+} \underset{(A .4)}{<}-2 \Omega^{+} \\
& \underset{(A .4)}{<}-\Omega^{+}+(K E)^{+} w_{k+k_{0}}^{+} \\
& \underset{(A .4)}{<}-\Delta_{u}^{+} \underline{1}-\Omega^{+}+\Delta_{u}^{+} \underline{1} \\
& +(K E)^{+} w_{k+k_{0}}^{+} \\
& \underset{(A .4)}{<} \quad g_{k+k_{0}+1}^{+}=g_{k+k_{0}}^{+}+\Delta_{u}^{+} \underline{1} \\
& +(K E)^{+} w_{k+k_{0}}^{+} \\
& \underset{(A .4)}{<} \Delta_{u}^{+} \underline{1}+\Omega^{+}
\end{aligned}
$$

The case $g_{k+k_{0}+1}^{+}<0$ and $g_{k+k_{0}+1}^{+} \notin G^{+}$for $g_{k}^{+}$, $g_{k+k_{0}}^{+}>\Delta_{u}^{+} \underline{1}+\Omega^{+}$is not possible since

$$
\begin{aligned}
g_{k+k_{0}+1}^{+} & =g_{k+k_{0}}^{+}-\Delta_{u}^{+} \underline{1} \\
+ & (K E)^{+} w_{k+k_{0}}^{+} \\
& >\Omega^{+}+(K E)^{+} w_{k+k_{0}}^{+}>0
\end{aligned}
$$

Similarly, the case $g_{k+k_{0}+1}^{+}>0$ and $g_{k+k_{0}+1}^{+} \notin G^{+}$ for $g_{k}^{+}, g_{k+k_{0}}^{+}<-\Delta_{u}^{+} \underline{1}-\Omega^{+}$cannot happen as

$$
\begin{aligned}
g_{k+k_{0}+1}^{+} & =g_{k+k_{0}}^{+}+\Delta_{u}^{+} \underline{1} \\
& +(K E)^{+} w_{k+k_{0}}^{+} \\
& <-\Omega^{+}+(K E)^{+} w_{k+k_{0}}^{+}<0 .
\end{aligned}
$$

Therefore, we have proven that $g_{k+k_{0}+1}^{+} \in G^{+}$and, by induction, the latter can be generalized to

$$
g_{k+k_{0}+m}^{+} \in G^{+}
$$

for every $m>0$. The sign of $g_{k}$ may change at each time step, causing the chattering in that way, but $g_{k}$ will stay in $G^{+}$. Having demonstrated that eq. (A.23) is satisfied if eq. (A.4) is valid, the proof ends.

\section{Appendix B \\ Proof of Theorem 3.2}

Assume that $g_{k} \notin G$. Then, eq. (22) becomes eq. (17) and the proof is similar to the one discussed in Appendix A. This means that $g_{k+k_{0}} \in G$ where $k_{0}$ is determined by eq. (A.18). Let $g_{k}^{j}$ be the $j^{t h}$ element of $g_{k}$ and assume that corresponding element $\left(K E w_{k+k_{0}}\right)^{j}<0$. Then

$$
\begin{aligned}
g_{k+k_{0}+1}^{j} & =g_{k+k_{0}}^{j}-\delta_{u}^{j}-\left|\left(K E w_{k+k_{0}}\right)^{j}\right| \\
& <\Omega^{j}-\left|\left(K E w_{k+k_{0}}\right)^{j}\right| \\
& <\delta_{u}^{j}-\left|\left(K E w_{k+k_{0}}\right)^{j}\right| \\
& <\delta_{u}^{j}
\end{aligned}
$$

where $\delta_{u}^{j}$ and $\Omega^{j}$ are the $j^{t h}$ elements in the diagonal of $\Delta_{u}$ and in vector $\Omega$, respectively . Then, from eqs. (B.1) and (22) we have

$$
g_{k+k_{0}+1}^{j}=\left(K E w_{k+k_{0}}\right)^{j} \in G^{j}
$$

If $\left(K E w_{k+k_{0}}\right)^{j}>0, g_{k+i}^{j}$ will continue to decrease and, after $k_{1}$ time instants

$$
\begin{aligned}
& k_{1}=\operatorname{int}\left(\left(\delta_{u}^{j}+\Omega^{j}\right)^{-1}\left(\delta_{u}^{j}-\Omega^{j}\right)\right)+1 \\
& g_{k+k_{0}+k_{1}}^{j} \in\left\{g_{k+i}^{j}:\right.\left.-\delta_{u}^{j}-\Omega^{j}<g_{k+i}^{j}<0\right\} \text { and } \\
& g_{k+k_{0}+k_{1}+1}^{j}=g_{k+k_{0}+k_{1}}^{j}+\delta_{u}^{j} \\
&+\left|\left(K E w_{k+k_{0}+k_{1}}\right)^{j}\right| \\
&>-\Omega^{j}+\left|\left(K E w_{k+k_{0}+k_{1}}\right)^{j}\right| \\
&>-\delta_{u}^{j}+\left|\left(K E w_{k+k_{0}+k_{1}}\right)^{j}\right| \\
&>-\delta_{u}^{j}
\end{aligned}
$$


Meanwhile, if $K E w_{i}<0$ for some $i>k+k_{0}$ then eqs. (B.1) and (B.2) stand. It is implied by eqs. (B.4) and

(22) that, from $i=k_{0}+k_{1}$

$$
g_{k+k_{0}+k_{1}+1}^{j}=\left(K E w_{k+k_{0}+k_{1}}\right)^{j} \in G^{j}
$$

From eqs. (B.2) and (B.5) we have that once $g_{k}$ enters $G$, it will stay in it, i.e.

$$
g_{k+i+1}=K E w_{k+i} \in G
$$

and, therefore, there is no chattering in sliding mode.

\section{References}

Bemporad, A. and Morari, M. Control of systems integrating logic, dynamics, and constraints. Automatica, 1999. 35(3):407 - 427. doi:10.1016/S00051098(98)00178-2.

Bemporad, A., Morari, M., Dua, V., and Pistikopoulos, E. N. The explicit linear quadratic regulator for constrained systems. Automatica, 2002. 38:3-20. doi:10.1016/S0005-1098(01)00174-1.

Benattia, S. E., Tebbani, S., and Dumur, D. Hierarchical control strategy based on robust mpc and integral sliding mode - application to a continuous photobioreactor. In Proceedings of 5th IFAC Conference on Nonlinear Model Predictive Control. pages 212-217, 2015. doi:10.1016/j.ifacol.2015.11.285.

Corradini, M. L. and Orlando, G. A vsc algorithm based on generalized predictive control. Automatica, 1997. 33(5):927-932. doi:10.1016/S00051098(96)00229-4.

Furuta, K. Sliding mode control of a discrete system. Systems \& Control Letters, 1990. 14(2):145152. doi:10.1016/0167-6911(90)90030-X.

Garcia-Gabin, W., Zambrano, D., and Camacho, E. F. Sliding mode predictive control of a solar air conditioning plant. Control Engineering Practice, 2009. 17(6):652-663. doi:10.1016/j.conengprac.2008.10.015.

Golo, G. and Milosavljević, C. Robust discrete-time chattering free sliding mode control. Systems \& Control Letters, 2000. 41(1):19-28. doi:10.1016/S01676911(00)00033-5.

Incremona, G. P., Ferrara, A., and Magni, L. Hierarchical model predictive/sliding mode control of nonlinear constrained uncertain systems. In Proceedings of 5th IFAC Conference on Nonlinear Model Predictive Control. pages 102-109, 2015. doi:10.1016/j.ifacol.2015.11.268.
Inteco. Modular Servo System - Manual. Inteco, 2011.

Mignone, D. The REALLY BIG Collection of Logic Propositions and Linear Inequalities. Technical report, 2001. URL http://control.ee.ethz.ch/index.cgi?action= details;id=377; page=publications.

Milosavljević, C. General conditions for the existence of a quasisliding mode on the switching hyperplane in discrete variable structure systems. Automation and Remote Control, 1985. 46(3):307-314.

Milosavljević, C. Discrete-time vss. In A. Sabanović, L. Fridman, and S. Spurgeon, editors, Variable Structure Systems: from Principles to Implementation, chapter 5, pages 99-128. The Institution of Engineering and Technology, London, 2004. doi:10.1049/PBCE066E.

Mitić, D., Spasić, M., Hovd, M., and Antić, D. An approach to design of sliding mode based generalized predictive control. In Proceedings of IEEE 8th International Symposium on Applied Computational Intelligence and Informatics (SACI) 2013. pages 347351, 2013. doi:10.1109/SACI.2013.6608996.

Neelakantan, V. A. Modeling, design, testing and control of a two-stage actuation mechanism using piezoelectric actuators for automotive applications. Ph.D. thesis, Ohio State University, Department of Mechanical Engineering, 2005.

Qin, S. J. and Badgwell, T. A. A survey of industrial model predictive control technology. Control Engineering Practice, 2003. pages 733-764. doi:10.1016/S0967-0661(02)00186-7.

Raković, S. V., Kerrigan, E. C., Kouramas, K. I., and Mayne, D. Q. Invariant approximations of the minimal robustly positively invariant sets. IEEE Transactions on Automatic Control, 2005. pages 406 410. doi:10.1109/TAC.2005.843854.

Rawlings, J. B. and Mayne, D. Q. Model Predictive Control: Theory and Design. Nob Hill Publishing, Madison, Wisconsin, USA, 2009.

Rubagotti, M., Castanos, F., Ferrara, A., and Fridman, L. Integral sliding mode control for nonlinear systems with matched and unmatched perturbations. IEEE Transactions on Automatic Control, 2011. 56(11):2699-2704. doi:10.1109/TAC.2011.2159420.

Su, W.-C., Drakunov, S. V., and Ozguner, U. An o(t2) boundary layer in sliding mode for sampled-data systems. IEEE Transactions on Automatic Control, 2000. 45(3):482-485. doi:10.1109/9.847728. 
Utkin, V. I. Sliding Modes and Their Applications in Variable Structure Systems. MIR, Moscow, USSR, 1978.

Young, K. D., Utkin, V. I., and Özgüner, U. A control engineer's guide to sliding mode control. IEEE Transactions on Control Systems Technology, 1999.
Transactions on Control Systems Technology, 1999. 7(3):328-342. doi:10.1109/87.761053.

Yu, X. and Kaynak, O. Sliding-mode control with soft computing: A survey. IEEE Transactions on Industrial Electronics, 2009. 56(9):3275-3285. doi:10.1109/TIE.2009.2027531. 